\title{
The Association between Taking Dietary Supplements and Healthy Habits among Korean Adults: Results from the Fifth Korea National Health and Nutritional Examination Survey (2010-2012)
}

\author{
Jin-Wook Kim', So-Hye Lee', Jung-Eun Kim², Kyung-Do Han³, Tae-Eung Kwack', Bo-Seon Kim', Jeong-Eun Kim', \\ Eun-Bae Jo', Young-Kyu Park', Kyung-Shik Lee ${ }^{1, *}$ \\ 'Department of Family Medicine, Bundang Jesaeng Hospital, Seongnam, Korea \\ ${ }^{2}$ Department of Family Medicine, Incheon St. Mary's Hospital, The Catholic University of Korea College of Medicine, Incheon, Korea \\ ${ }^{3}$ Department of Biostatistics, The Catholic University of Korea College of Medicine, Seoul, Korea
}

Background: Recently, the number of people interested in health in South Korea has increased, and the rate of dietary supplement use is rising. Researchers have hypothesized that the rate of practicing healthy habits is higher among those who use dietary supplements than those who do not. Therefore, this study aimed to discover the association between taking dietary supplements and practicing various healthy habits in the Korean, adult population.

Methods: The sample included 15,789 adults over 19 years old who participated in the fifth Korea National Health and Nutrition Examination Survey. The user group was defined as those taking dietary supplements for more than 2 weeks during the previous year or once during the past month. Measures for the seven healthy habits were based on those included in the Alameda study and were analyzed accounting for the complex sampling design.

Results: The rate of taking dietary supplements was significantly higher in women, middle aged participants, urban residents, those with a higher income, those with a higher education level, and nonsmokers as well as among women with a moderate subjective health status, women who limited their alcohol content, and women with dyslipidemia. In the adjusted analysis, the rate of performing three of the 'Alameda 7' habits-eating breakfast regularly, restricting snacking, and limiting drinking - was higher in the female dietary supplement user group than in the other groups. Women practiced more healthy habits and had a higher dietary supplement intake rate than men.

Conclusion: We found that taking dietary supplements in Korean adults is highly associated with demographic and social factors. Taking dietary supplements had a relationship with dietary habits, and there was no significant association between dietary supplement and other healthy habits. Thus in the health clinic, we suggest that taking dietary supplements complements a patient's healthy habits, with the exception of dietary habits, for health promotion.

Keywords: Dietary Supplements; Health Behavior; Health Promotion 


\section{INTRODUCTION}

In South Korea, the number of people interested in health has increased following recent advancements in medicine and improvements in economic status. In addition, research has shown that an individual's health status is more dependent upon one's health behavior than upon the health care system. ${ }^{1)}$ Health behavior is defined as one's voluntary actions carried out in order to prevent or discover a disease when one does not have any symptoms and includes healthy habits such as regular exercise and maintaining a balanced diet. ${ }^{1)}$ In a prospective study conducted in Alameda County by Belloc and Breslow ${ }^{2)}$ and Belloc, ${ }^{3)}$ seven healthy habits, including regular exercise, being a nonsmoker, moderate drinking or abstinence from drinking, getting adequate sleep, eating breakfast regularly, and limiting snacking were assessed to examine their association with the mortality rate among participants. According to the study, there was a negative correlation between these healthy habits and age-adjusted mortality, findings that are supported by many other studies. ${ }^{4,5}$ In Korea, several domestic studies promoting health based on the healthy habits presented in the Alameda study have been conducted, ${ }^{6}$ including studies such as the Korea National Health and Nutrition Examination Survey.

According to the 2005 National Health and Nutrition Examination Survey, approximately $30 \%$ of South Korean adults were taking dietary supplements. This indicates that South Korean adults recognize that taking dietary supplements is an important healthy habit. Given that dietary supplements are associated with both personal health and increases in societal health care costs, it is important to define the rate of dietary supplement intake. In addition, the cross-reaction of dietary supplements with prescription medicines can have both direct and indirect effects on medical treatments. ${ }^{7}$ According to the Korean National Statistical Office, the rate of dietary supplement intake is expected to continue to increase; ${ }^{8)}$ however, large-scale studies with representative data in relation to healthy dietary supplement intake in a Korean population are rare. ${ }^{9)}$

In this study, it was hypothesized that dietary supplement intake was associated with numerous healthy habits and that the dietary supplement user group would have a higher rate of practicing healthy habits than the group not taking supplements (nonuser group). This study also examined the hypothesis that the more an individual practiced healthy habits, the higher the rate of dietary supplement use would be. Hence, this study aimed to evaluate the association between dietary supplement use and healthy habits among the entire adult population in Korea using data from the fifth National Health and Nutrition Examination Survey.

\section{METHODS}

\section{Study Period and Participants}

The Korea National Health and Nutrition Examination Survey is a cohort survey ${ }^{10)}$ investigating the health behavior, chronic disease prevalence, and food and nutrition intake status of the Korean population.
The survey receives institutional review board approval from the Korea Centers for Disease Control and Prevention. ${ }^{11)}$ Selected participants visit a mobile screening clinic to complete the general examination and health survey. All participants provided informed consent prior to completing the surveys. Nutritionists conduct nutrition surveys during household visits within 1 week following the visit to the screening clinic. $^{11)}$ This study analyzed the data of participants $>19$ years old who completed the fifth National Health and Nutrition Examination Survey conducted from January 2010 to December 2012. The target population for this analysis was the entire adult population of South Korea. Of the 25,534 eligible participants, 5,935 under the age of 19 years and 3,810 with missing data were eventually excluded from the analysis, leaving 15,789 participants (6,331 men and 9,458 women) in the final analytic sample.

\section{Study Methods}

Demographic characteristics, health behaviors, and disease history were investigated using the health questionnaire. Age was categorized into three groups: 19-40 years old, 40-65 years old, and $>65$ years old. Those who had a spouse or were living with someone were classified as married, and all others were classified as unmarried. Education level was divided into four categories: less than 7 years (below elementary school graduation), more than 7 years but less than 12 years (beyond elementary school graduation but below high school graduation), more than 12 years but less than 16 years (beyond high school graduation), and more than 16 years (beyond college graduation). Monthly household income was classified into four quartiles based on the total population income (low, lower middle, higher middle, and high), and region of residence was classified as urban or nonurban. Subjective health status was divided into five categories using a Likert scale (very good, good, moderate, bad, and very bad). In accordance with World Health Organization recommendations, the high-risk drinking group included those with an average per session alcohol consumption of more than $61 \mathrm{~g}$ for men and more than $41 \mathrm{~g}$ for women; those who drank less than this were classified into the non-high-risk drinking group. Smoking was classified using three groups: those who had no smoking experience, past smokers, and current smokers. The presence of a lifestyle-associated chronic disease was indicated if the participant reported having been diagnosed with diabetes, hypertension, dyslipidemia, or osteoarthritis. Physical examinations and blood tests were performed by the survey team to assess obesity and metabolic syndrome. Using standardized instruments, the team obtained the height, weight, waist circumference, blood pressure, fasting blood glucose levels, fasting triglycerides levels, and fasting blood cholesterol concentrations of participants. The body mass index (BMI) was calculated by dividing the measured weight by the square of the height, and obesity was defined as having a BMI $>25 \mathrm{~kg} / \mathrm{m}^{2} .{ }^{12)}$ Metabolic syndrome was indicated in participants who satisfied at least three of the following five criteria: (1) a waist circumference $>90 \mathrm{~cm}$ for men and $>80 \mathrm{~cm}$ for women; (2) fasting blood glucose $\geq 100 \mathrm{mg} / \mathrm{dL}$ or an increase in blood glucose even with medication; (3) fasting serum triglycerides 
$\geq 150 \mathrm{mg} / \mathrm{dL}$ or the use of cholesterol lowering medication; (4) serum high density lipoprotein cholesterol level $<40 \mathrm{mg} / \mathrm{dL}$ for men and $<50$ $\mathrm{mg} / \mathrm{dL}$ for women or the use of cholesterol-lowering medication; or (5) systolic blood pressure $>130 \mathrm{~mm}$ Hg or diastolic blood pressure $>85$ $\mathrm{mm} \mathrm{Hg}$ or the use of antihypertensive drugs for patients with a history of hypertension. ${ }^{13)}$

Dietary supplement usage was assessed using the nutrition survey conducted by a team of nutritionists. ${ }^{11)}$ Frequent dietary supplement users were defined as those who had taken vitamins, minerals, or health functional foods for more than 2 weeks in the past year or more than once in the past month. Participants who had never taken supplements were identified as nonusers. Questions regarding healthy habits were assessed via the health survey through interviews conducted by the researchers. The questions included on the National Health and Nutrition Examination Survey were created in accordance with the Alameda study's seven healthy habits. Participants who engaged in the seven healthy habits were categorized as healthy habit practitioners, and those who did not were categorized as nonpractitioners. Nonpractitioners made up the control group for this study.

Adequate sleep was defined as obtaining 7 to 8 hours of sleep. Eating breakfast regularly was defined as having eaten breakfast on both of the previous 2 days. Although habitual snacking was assessed in the fourth survey, ${ }^{14)}$ it was deleted from the fifth survey. Therefore, a question regarding voluntary weight control, indicated by a commitment to try to maintain or reduce weight by adjusting diet instead of skipping meals in the past year, was included to compensate. A normal BMI was defined as between $18 \mathrm{~kg} / \mathrm{m}^{2}$ and $23 \mathrm{~kg} / \mathrm{m}^{2}$. High-intensity physical activity was defined as participation in running, climbing, highspeed cycling, fast swimming, soccer, basketball, jump rope, squash, tennis, or professional and athletic activities (such as moving heavy items around) for more than 10 minutes a day, more than 3 times a week. High-risk drinking was defined as drinking more than the average consumption per session (as defined by the World Health Organization), and smoking was categorized as no smoking, past smoking, or current smoking. Participants who engaged in these healthy habits were considered healthy habit practitioners.

\section{Statistical Analysis}

The National Health and Nutrition Examination Survey is a sample survey that uses a complex sample design to estimate the rate of behaviors of the target South Korean adult population. All analyses were performed using sample weights in SAS Survey Procedure ver. 9.3 (SAS Institute Inc., Chicago, IL, USA) ${ }^{15)}$ All data were classified by sex, and chi-square tests were performed based on sociodemographic characteristics of the dietary supplement users. Using a logistic regression analysis, unadjusted odds ratios (ORs) for the seven healthy habits of dietary supplement users and nonusers were calculated. Adjusted ORs controlling for social and economic factors including age, marital status, income level, level of education, area of residence, subjective health status, chronic diseases, and disease history were also calculated. Additionally, a health index score was calculated by giving one point for each healthy habit practiced. The score ranged from zero to seven, and the trend for dietary supplements use was described as ' $\mathrm{P}$ for trend.'

\section{RESULTS}

The fifth National Health and Nutrition Examination Survey found that $45.96 \%$ of South Korean adults 19 years and older were taking dietary supplements. Women had higher usage than men, and both men and women had higher usage during middle age. Dietary supplement usage was significantly higher amongst those who lived in an urban region, those with a higher level of education, and those with a higher monthly household income. Both men and women who considered their health status to be normal showed a usage rate of approximately $50 \%$, but this was statistically significant among women only. Both men and women nonsmokers exhibited high dietary supplement usage rates; however, among men, current smokers had a higher rate of dietary supplement use (49.2\%) than both nonsmokers (31.9\%) and past smokers (18.9\%). Both male and female high-risk drinkers had low dietary supplement usage rates, but this was statistically significant among women only. Dietary supplement usage was lower in patients with a chronic disease when compared to those without a history of disease, but the difference was only significant in women with diabetes. However, women with dyslipidemia showed a high usage rate (Table 1).

When the relationship between dietary supplement usage and healthy habits was adjusted for age, socioeconomic factors, history of osteoarthritis, and history of metabolic syndrome, the rate of eating breakfast regularly was $19 \%$ higher in men (OR, 1.19; 95\% confidence interval [CI], 1.01 to 1.4 ) and $43 \%$ higher in women (OR, 1.43; $95 \% \mathrm{CI}$, 1.25 to 1.64) in the dietary supplement user group compared to the nonuser group. Similarly, the rate of weight control through snack restriction was $18 \%$ higher in men (OR, 1.18; 95\% CI, 1.01 to 1.39) and $16 \%$ higher in women (OR, 1.16; 95\% CI, 1.03 to 1.3 ) in the dietary supplement user group compared to the nonuser group. In addition, women in the dietary supplement user group were more likely to limit alcohol consumption (Table 2).

Furthermore, the health index score indicated that there was a significant positive correlation ( $\mathrm{P}$ for trend $<0.001$ ) between dietary supplement usage and the health index score among women. In men, supplement usage was higher in those who practiced at least one healthy habit than in those who did not practice any, but this was not statistically significant ( $\mathrm{P}$ for trend=0.19) (Figure 1).

\section{DISCUSSION}

Dietary supplement usage was higher in women, middle-aged participants, urban residents, and nonsmokers as well as those with a higher level of education and those with a high income. The rate of dietary supplement usage was significantly higher in women who did not engage in high-risk drinking, women who subjectively considered their 
Table 1. Characteristics of Korean adults taking dietary supplements

\begin{tabular}{|c|c|c|c|c|c|c|}
\hline \multirow{2}{*}{ Characteristic } & \multicolumn{3}{|c|}{ Male } & \multicolumn{3}{|c|}{ Female } \\
\hline & User & Nonuser & P-value & User & Nonuser & P-value \\
\hline Number* & 2,458 & 3,873 & & 4,799 & 4,799 & \\
\hline Age (y) & & & 0.001 & & & $<0.001$ \\
\hline 19-39 & $586(38.4)$ & $1,101(43.6)$ & & $1,310(34.8)$ & $1,551(42.2)$ & \\
\hline $40-60$ & $1,183(49.0)$ & $1,741(45.3)$ & & $2,418(50.7)$ & $1,894(40.1)$ & \\
\hline$>60$ & $689(12.7)$ & $1,031(11.0)$ & & $1,071(14.5)$ & $1,214(17.7)$ & \\
\hline Marital status & $276(22.1)$ & 635 (29.3) & $<0.0001$ & $430(13.7)$ & $640(21.4)$ & $<0.0001$ \\
\hline Education level (y) & & & $<0.0001$ & & & $<0.0001$ \\
\hline$<7$ & $371(9.8)$ & 841 (13.2) & & $1,345(21.8)$ & 1,694 (28.3) & \\
\hline$\geq 7,<12$ & $295(9.3)$ & 487 (10.2) & & $546(10.9)$ & $418(8.6)$ & \\
\hline$\geq 12,<16$ & $823(38.9)$ & $1395(43.8)$ & & $1,491(34.5)$ & $1,444(37.5)$ & \\
\hline$\geq 16$ & $963(42.0)$ & $1143(32.8)$ & & $1,411(32.7)$ & $1,092(25.5)$ & \\
\hline Monthly income & & & $<0.0001$ & & & $<0.0001$ \\
\hline Low & $364(10.6)$ & 836 (15.4) & & $809(14.0)$ & $1,177(21.5)$ & \\
\hline Lower middle & $601(24.8)$ & $1,031(28.4)$ & & $1,186(26.7)$ & $1,217(28.2)$ & \\
\hline Higher middle & $683(29.8)$ & $1,063(31.3)$ & & $1,308(28.9)$ & $1,184(27.7)$ & \\
\hline High & 788 (34.8) & 907 (24.8) & & $1,442(30.5)$ & $1,042(22.6)$ & \\
\hline Urban resident & $1,997(83.6)$ & $2,899(77.6)$ & $<0.0001$ & $3,989(84.0)$ & $3,501(78.0)$ & $<0.0001$ \\
\hline Self-reported health status & & & 0.40 & & & 0.01 \\
\hline Very good & $135(5.5)$ & $209(5.5)$ & & $164(3.6)$ & $194(4.5)$ & \\
\hline Good & $819(34.9)$ & $1,191(33.0)$ & & $1,271(26.2)$ & $1,205(26.9)$ & \\
\hline Moderate & $1,144(46.9)$ & $1,774(46.9)$ & & $2,345(50.0)$ & $2,132(46.8)$ & \\
\hline Bad & $299(11.2)$ & $576(12.8)$ & & $834(17.1)$ & $876(17.6)$ & \\
\hline Very bad & $55(1.5)$ & $117(1.8)$ & & $182(3.1)$ & $243(4.2)$ & \\
\hline Smoking status & & & $<0.0001$ & & & 0.04 \\
\hline Nonsmoker & 537 (31.9) & $832(30.5)$ & & $4,405(92.8)$ & $4,244(91.4)$ & \\
\hline Ex-smoker & $421(18.9)$ & $556(13.8)$ & & $102(2.8)$ & $92(2.5)$ & \\
\hline Current smoker & $770(49.2)$ & $1,549(55.7)$ & & $168(4.5)$ & $217(6.0)$ & \\
\hline High-risk drinking & $412(24.4)$ & $753(25.1)$ & 0.65 & $144(5.6)$ & $168(7.5)$ & 0.03 \\
\hline Obesity & $822(35.4)$ & $1,360(36.3)$ & 0.53 & $1,358(27.3)$ & $1,435(28.9)$ & 0.18 \\
\hline Diabetes mellitus & $283(8.9)$ & $493(9.7)$ & 0.37 & $355(6.6)$ & $412(8.0)$ & 0.04 \\
\hline Hypertension & $952(31.4)$ & $1,464(30.1)$ & 0.39 & $1,435(24.1)$ & $1,430(24.2)$ & 0.90 \\
\hline Dyslipidemia & $316(11.9)$ & 431 (10.7) & 0.21 & 825 (15.2) & $622(12.0)$ & 0.0001 \\
\hline Metabolic syndrome & 712 (25.3) & $1,070(25.3)$ & 0.99 & $1,332(25.5)$ & $1,336(26.1)$ & 0.56 \\
\hline Osteoarthritis & $123(3.3)$ & $185(2.9)$ & 0.36 & $858(13.7)$ & 758 (12.4) & 0.10 \\
\hline
\end{tabular}

Values are presented as weighted mean ( \pm standard error) for continuous variables and unweighted percentages for categorical variables. All variables were categorized based on sex.

*Number of participants who responded to questions regarding taking dietary supplements on the fifth Korea National Health and Nutrition Examination Survey (2010-2012).

Table 2. ORs and 95\% Cls for the relationship between taking dietary supplements and seven healthy habits in Korean adults

\begin{tabular}{|c|c|c|c|c|}
\hline \multirow{2}{*}{ Healthy habits } & \multicolumn{2}{|c|}{ Male } & \multicolumn{2}{|c|}{ Female } \\
\hline & Crude $\mathrm{OR}^{\star}(95 \% \mathrm{Cl})$ & Adjusted OR (95\% Cl) & Crude OR (95\% Cl) & Adjusted OR (95\% Cl) \\
\hline Adequate sleep (7-8 h/d) & $0.94(0.83-1.07)$ & $0.93(0.81-1.07)$ & $1.04(0.94-1.16)$ & $1.04(0.94-1.16)$ \\
\hline Regular breakfast & $1.17(1.00-1.36)$ & $1.19(1.01-1.4)$ & $1.45(1.27-1.65)$ & $1.43(1.25-1.64)$ \\
\hline Weight control by snack restriction & $1.18(1.02-1.38)$ & $1.18(1.01-1.39)$ & $1.18(1.06-1.32)$ & $1.16(1.03-1.3)$ \\
\hline $18 \mathrm{~kg} / \mathrm{m}^{2}<\mathrm{BMl}<23 \mathrm{~kg} / \mathrm{m}^{2}$ & $0.97(0.84-1.11)$ & $0.97(0.84-1.13)$ & $1.05(0.95-1.16)$ & $1.043(0.94-1.15)$ \\
\hline Regular exercise & $1.23(0.96-1.58)$ & $1.16(0.89-1.52)$ & $1.14(0.89-1.45)$ & $1.173(0.92-1.5)$ \\
\hline Non-high-risk alcohol use & $1.08(0.94-1.25)$ & $1.09(0.94-1.26)$ & $1.31(1.1-1.54)$ & $1.30(1.1-1.54)$ \\
\hline Nonsmoker & $0.9(0.76-1.05)$ & $0.88(0.75-1.04)$ & $1.14(0.97-1.34)$ & $1.071(0.9-1.28)$ \\
\hline
\end{tabular}

The seven healthy habits were based on those included in the Alameda study. Adequate sleep was defined as 7-8 hours per day. Regular breakfast was defined as eating breakfast on 2 sequential days. Weight control was defined as the control of weight by restricting snacks. Normal BMl was defined as $>18 \mathrm{~kg} / \mathrm{m}^{2}$ and $<23 \mathrm{~kg} / \mathrm{m}^{2}$. Regular exercise was defined as high intensity physical activity such as running, mountain climbing, tennis, soccer, or baseball for more than 10 minutes a day at least 3 times per week. Non-high-risk alcohol use was defined as alcohol consumption less than $61 \mathrm{~g}$ in men and $41 \mathrm{~g}$ in women in accordance with World Health Organization recommendations. A nonsmoker was defined as a person who did not smoke at all.

$\mathrm{OR}$, odds ratio; $\mathrm{Cl}$, confidence interval; $\mathrm{BMI}$, body mass index.

*Crude ORs were based on logistic regression analysis. 


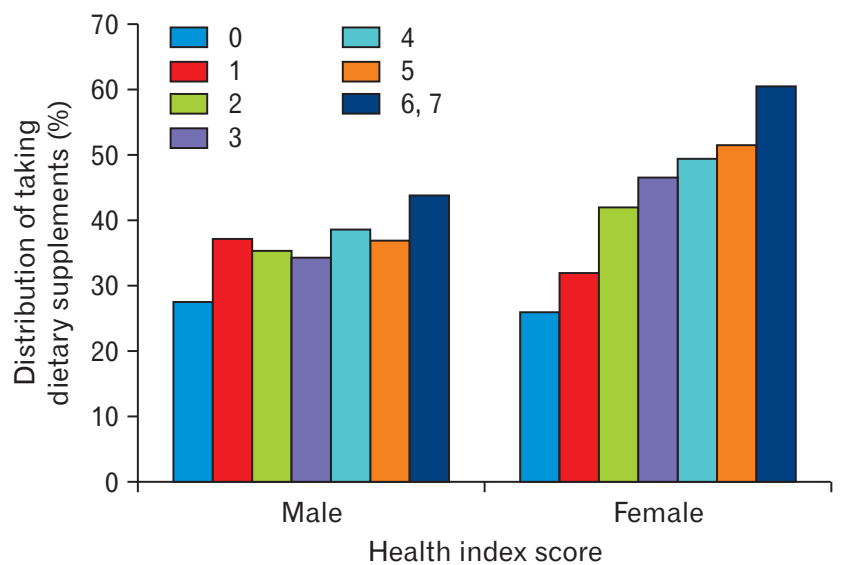

Figure 1. The distribution of dietary supplement intake by the health index score* in Korean Adults. ${ }^{*}$ The health index score was calculated based on the presence of the seven healthy habits. One point was given for each of the seven healthy habits resulting in a score from zero to seven. The seven healthy habits are adequate sleep, eating breakfast regularly, weight control through snack restriction, a body mass index between $18 \mathrm{~kg} / \mathrm{m}^{2}$ and $23 \mathrm{~kg} / \mathrm{m}^{2}$, regular exercise, non-high-risk alcohol use, and being a nonsmoker. The $\mathrm{P}$ for trend was calculated (male $\mathrm{P}$ for trend $=0.19$, female $\mathrm{P}$ for trend $<0.001$ ).

health to be normal, and women with dyslipidemia. In addition, there was a significant positive correlation between the number of practiced healthy habits and the rate of dietary supplement use in women. This study also found that both male and female participants who defined their subjective health status as normal also had high dietary supplement usage, a finding that has not been observed in previous studies. However, trends for dietary supplement use and area of residence, level of education, and household income were consistent with the results seen in other studies. ${ }^{6,13)}$ The results also coincided with studies conducted in America and Japan that showed higher dietary supplement usage in households with a stable socioeconomic status. ${ }^{16-18)} \mathrm{Be}-$ cause these factors impact the rate of dietary supplement usage, they were included in the adjusted analysis regarding the association between dietary supplement usage and healthy habits.

After adjustment, it was found that both male and female dietary supplement users were more likely to practice regular breakfast eating habits and had higher rates of maintaining and regulating their weight through proper diet and snack restriction than did nonusers. However, only women in the dietary supplement user group were more likely to limit their alcohol consumption. These healthy habits that taking regular breakfast, proper diet with snack restriction, and alcohol consumption limitation are related to dietary habits. People who use dietary supplements may be more likely to practice healthy habits because they consider dietary supplements to be a part of a diet that promotes health. Similar results were seen by Belloc and Breslow ${ }^{2)}$ and Belloc ${ }^{3)}$ who showed that snack restriction resulted in slightly, but significantly, better physical health. However, with regard to sleeping, exercising, and smoking, the results showed that people who used dietary supplements actually had about the same or an even lower possibility of practicing healthy habits. This contradicts the hypothesis that people who use dietary supplements practice more healthy habits because they are more interested in promoting overall health. Chiou et al. ${ }^{19}$ found a similar paradoxical effect in a cohort study comparing vitamin and placebo groups. The authors showed that participants who believed that they were taking vitamins were actually neglecting to maintain their health, and there was no association between dietary supplement intake and improvement in health. Chiou et al. ${ }^{19)}$ argued that the vitamin group believed that taking a dietary supplement would be enough in itself to promote their health, and thus actually made fewer efforts to change their behavior. ${ }^{20)}$ According to the South Korean National Statistical Office, dietary supplement use is steadily increasing in South Korea (as in the United States and Taiwan) ${ }^{8)}$ but the results of the current study indicate that this may not necessarily be related to individual health promotion. Furthermore, domestic research, as well as research conducted in the United States and Japan, has shown no significant relationship between dietary supplement usage and sleep habits, results that are supported by this study. However, this study found that the proportion of smokers who used dietary supplements was higher than that of smokers who did not use dietary supplements, results that contradict previously conducted domestic research.

This study has several limitations. First, this study did not take into account differences regarding the amount or method of exercise. Since the Alameda study was conducted with a population of runners, the sample for this study was also selected from participants who participated in high-intensity physical exercise (e.g., running not walking or jogging). However, it can be difficult for older adults to participate in high-intensity exercises due to diseases such as arthritis. Nonetheless, we did not adjusted for differences in exercise intensity in the present study. Second, the definition of smoking and high-risk drinking differed slightly between this study, the Alameda study, and other studies. Even though this study and the Alameda study both defined participants with no smoking experience as healthy habit practitioners, past smokers who have not smoked for a long period or current smokers who have an interest in quitting may also have an interest in their health, which may affect their dietary supplement use. In the Alameda study, alcohol intake was measured using the past 2 weeks; however, this study did not measure average alcohol intake, so high-risk drinking was defined as drinking more than the average recommended amount per session (as outlined by the World Health Organization). Finally, the questionnaire for this study did not examine the types of supplements nor the number of supplements taken per day. In the fourth survey, supplements were categorized as vitamins or minerals, multivitamins, and health functional foods. ${ }^{7)}$ The fourth survey also covered the length of the period in which the supplements were taken, the number used daily, and the dosage per each use. ${ }^{7)}$ However, in the fifth survey, categorization was difficult because the questions were open-ended (i.e., participants wrote answers instead of making selections). Therefore, associations between diseases and the kinds of dietary supplements, the number consumed, and classification of health lifestyle are limited. Since measurement regarding dietary supplement intake is broad, more defined measures and categorization would al- 
low for more clear analyses regarding associations with health behaviors and, thus, more relevant implications.

Nevertheless, this was a large-scale study regarding the association between dietary supplement use, healthy habits, the risk of chronic diseases, and health promotion linked with age, socioeconomic factors, and healthy habits in the South Korean adult population. In addition, this study identified important associations regarding subjective health status and adequate sleep related to dietary supplements that had not been identified in previous studies. Future studies should include any missing variables and elaborate on the results of the present study. Future studies should also aim for institutionalized categorization and quality management. Furthermore, the misconception that one can ensure one's health simply by using dietary supplements should be corrected during health screening counseling and outpatient care, and dietary supplement users should be aided in complementing their dietary supplement intake by adopting healthy habits.

\section{CONFLICT OF INTEREST}

No potential conflict of interest relevant to this article was reported.

\section{REFERENCES}

1. The Korean Society for Preventive Medicine. Preventive medicine and public health. 2nd ed. Seoul: Gyechuk Munwhasa; 2013.

2. Belloc NB, Breslow L. Relationship of physical health status and health practices. Prev Med 1972;1:409-21.

3. Belloc NB. Relationship of health practices and mortality. Prev Med 1973;2:67-81.

4. Breslow L, Enstrom JE. Persistence of health habits and their relationship to mortality. Prev Med 1980;9:469-83.

5. Schoenborn CA. Health habits of U.S. adults, 1985: the "Alameda 7" revisited. Public Health Rep 1986;101:571-80.

6. Kwak TH, Jee SH, Nam CM, Lee SY. The relationship between seven health habits (Alameda 7) and health-related quality of life. J Korean Acad Fam Med 2005;26:337-45.

7. Yi HH, Park HA, Kang JH, Kang JH, Kim KW, Cho YG, et al. What types of dietary supplements are used in Korea?: data from the Korean National Health and Nutritional Examination Survey 2005. Korean J Fam Med 2009;30:934-43.

8. Statistics Korea. Taking dietary supplements experience rate trend in Korean people [Internet]. Daejeon: Statistics Korea; 1996 [cited 2015 May 27]. Available from: http://kosis.kr.
9. Korea Food and Drug Administration. Establishment of legislative system to control health functional food. Cheongju: Korea Food and Drug Administration; 2002.

10. Korea Centers for Disease Control and Prevention. The fifth Korea National Health and Nutrition Examination Survey (KNHANES V), 20102012. Cheongju: Korea Centers for Disease Control and Prevention; 2012.

11. Korea Centers for Disease Control and Prevention. Fact sheet: the fifth Korea National Health and Nutrition Examination Survey (KNHANES V), 2010-2012 survey conduct guideline [Internet]. Cheongju: Korea Centers for Disease Control and Prevention; 2012 [cited 2012 Sep 12]. Available from: https://knhanes.cdc.go.kr/knhanes/index.do.

12. The Korean Academy of Family Medicine. Textbook of family medicine. 4th ed. Paju: Koonja; 2013. p. 225.

13. Alberti KG, Eckel RH, Grundy SM, Zimmet PZ, Cleeman JI, Donato KA, et al. Harmonizing the metabolic syndrome: a joint interim statement of the International Diabetes Federation Task Force on Epidemiology and Prevention; National Heart, Lung, and Blood Institute; American Heart Association; World Heart Federation; International Atherosclerosis Society; and International Association for the Study of Obesity. Circulation 2009;120:1640-5.

14. Korea Centers for Disease Control and Prevention. The fourth Korea National Health and Nutrition Examination Survey (KNHANES IV), 2007-2009. Cheongju: Korea Centers for Disease Control and Prevention; 2009.

15. Joo YH, Hwang SH, Han KD, Seo JH, Kang JM. Relationship between olfactory dysfunction and suicidal ideation: the Korea National Health and Nutrition Examination Survey. Am J Rhinol Allergy 2015;29:26872.

16. Lee YO, Song YJ. Sociodemographic characteristics, lifestyle factors, and nutrient intake by taking vitamin/mineral supplements. Korean J Food Cult 2010;25:480-6.

17. Radimer K, Bindewald B, Hughes J, Ervin B, Swanson C, Picciano MF. Dietary supplement use by US adults: data from the National Health and Nutrition Examination Survey, 1999-2000. Am J Epidemiol 2004; 160:339-49.

18. Ishihara J, Sobue T, Yamamoto S, Sasaki S, Tsugane S; JPHC Study Group. Demographics, lifestyles, health characteristics, and dietary intake among dietary supplement users in Japan. Int J Epidemiol 2003; 32:546-53.

19. Chiou WB, Yang CC, Wan CS. Ironic effects of dietary supplementation: illusory invulnerability created by taking dietary supplements licenses health-risk behaviors. Psychol Sci 2011;22:1081-6.

20. Macreae F. Vitamins give people 'license to indulge.' The Telegraph (Health News). 2012 Dec 4. English. 